\title{
Dynamic Changes in the Nigrostriatal Pathway in the MPTP Mouse Model of Parkinson's Disease
}

\author{
Dongping Huang, ${ }^{1}$ Jing Xu, ${ }^{2}$ Jinghui Wang, ${ }^{1}$ Jiabin Tong, ${ }^{1}$ Xiaochen Bai, ${ }^{1}$ Heng Li, ${ }^{1}$ \\ Zishan Wang, ${ }^{1}$ Yulu Huang, ${ }^{3}$ Yufei Wu, ${ }^{3}$ Mei Yu, ${ }^{1}$ and Fang Huang ${ }^{1}$ \\ ${ }^{1}$ The State Key Laboratory of Medical Neurobiology, The Institutes of Brain Science and the Collaborative Innovation Center for Brain \\ Science, Shanghai Medical College, Fudan University, 138 Yixueyuan Road, Shanghai 200032, China \\ ${ }^{2}$ School of Life Science and Technology, Tongji University, 1239 Siping Road, Shanghai 200092, China \\ ${ }^{3}$ School of Basic Medical Sciences, Fudan University, 138 Yixueyuan Road, Shanghai 200032, China
}

Correspondence should be addressed to Mei Yu; yumei@fudan.edu.cn and Fang Huang; huangf@shmu.edu.cn

Received 8 March 2017; Revised 9 May 2017; Accepted 28 June 2017; Published 31 July 2017

Academic Editor: Ivan Bodis-Wollner

Copyright (C) 2017 Dongping Huang et al. This is an open access article distributed under the Creative Commons Attribution License, which permits unrestricted use, distribution, and reproduction in any medium, provided the original work is properly cited.

\begin{abstract}
The characteristic brain pathology and motor and nonmotor symptoms of Parkinson's disease (PD) are well established. However, the details regarding the causes of the disease and its course are much less clear. Animal models have significantly enriched our current understanding of the progression of this disease. Among various neurotoxin-based models of PD, 1-methyl-4-phenyl1,2,3,6-tetrahydropyridine (MPTP) mouse model is the most commonly studied model. Here, we provide an overview of the dynamic changes in the nigrostriatal pathway in the MPTP mouse model of PD. Pathophysiological events, such as reductions in the striatal dopamine (DA) concentrations and levels of the tyrosine hydroxylase (TH) protein, depletion of TH-positive nerve fibers, a decrease in the number of TH-positive neurons in the substantia nigra pars compacta (SNpc), and glial activation, are addressed. This article will assist with the development of interventions or therapeutic strategies for PD.
\end{abstract}

\section{Introduction}

Parkinson's disease (PD) is the second most common neurodegenerative disorder, which is prevalent among the elderly $[1,2]$. An anatomical circuit, the nigrostriatal pathway, is required for the fine tuning of basal ganglion function. This pathway is preferentially damaged in patients with $\mathrm{PD}$ and subsequently contributes to the clinical motor abnormalities, including tremor, muscle stiffness, a paucity of voluntary movements, and postural instability [3]. The main neuropathological features of $\mathrm{PD}$ are the loss of dopaminergic neurons in the substantia nigra pars compacta $(\mathrm{SNpc})$ and their projections into the caudate nucleus, as well as the cytoplasmic accumulation of proteinaceous aggregates, Lewy bodies (LBs), which were named after the neurologist Frederic Lewy [4]. During a 200-year period, the characteristic brain pathology and motor and nonmotor symptoms of PD have been well described. However, the etiology and pathogenesis of $\mathrm{PD}$ remain unclear $[5,6]$. Based on abundant studies of postmortem tissue and various genetic or neurotoxic animal models, the death of dopaminergic neurons has been linked to mitochondrial dysfunction, oxidative stress, neuroinflammation, and insufficient protein degradation [7]. Since the first synthesis of MPTP in the 1970s, MPTP-based animal models have greatly improved our understanding of the cause and course of the disease $[8,9]$. Here, we provide a brief review of the dynamic changes observed in the nigrostriatal pathway in the MPTP mouse model of PD.

\section{Microglia, Astrocytes, and Neurons in the Nigrostriatal Pathway}

Microglia and astrocytes are categorized as glial cell populations. In contrast to other glial cells and neurons, which are of neuroectodermal origin, microglia are derived from primitive hematopoiesis in the fetal yolk sac and migrate into the brain during early fetal development $[10,11]$. Using ablation approaches in the adult brains, microglia have been 
shown to possess a repopulation capacity; approximately 1 week after depletion, microglia are restored to their normal density [12-14]. Microglia constitute $5-20 \%$ of the total glial cell population in the rodent brains. The basal ganglia and substantia nigra (SN) are among the most densely populated areas [15]. Acting as primary cells that respond to pathogen infection and injury, microglial cells play the roles of resident macrophages in the central nervous system (CNS) $[10,16,17]$. By adopting an "amoeboid" phenotype, activated microglia produce many proinflammatory mediators, including cytokines, chemokines, reactive oxygen species (ROS), and nitric oxide (NO) [10]. The presence of reactive microglia in the SNpc of postmortem human brain tissue was first revealed as early as in 1988, suggesting the involvement of neuroinflammation in the pathogenesis of PD $[18,19]$.

Astrocytes represent the most abundant glial cell type in the adult brain [20]. The most important functions of astrocytes include the maintenance of water and ion homeostasis, participation in the tripartite synapse, and the formation of the blood brain barrier (BBB) [21]. Proteins that are generally used as astrocyte markers are the intermediate filament protein, glial fibrillary acidic protein (GFAP), the glutamate transporter, GLAST, and the aldehyde dehydrogenase 1 family member L1 (Aldh1L1) [22, 23]. Like microglia, astrocytes respond to inflammatory stimuli such as LPS, IL-1 $\beta$, and TNF- $\alpha$ by producing an array of pro- and anti-inflammatory mediators, antioxidants, and neurotrophic factors. Reactive astrogliosis, which is characterized by increased expression levels of GFAP and hypertrophy of the cell body and its extensions, has been observed in affected brain regions of patients with $\mathrm{PD}[24,25]$, indicating the involvement of astrocytes in the immune processes in PD [19].

Under physiological conditions, the crosstalk among neurons, astrocytes, and microglial cells are essential for the homeostasis of the nigrostriatal axis. Astrocytes promote the survival and maintenance of dopaminergic neurons by secreting various neurotrophic factors in the SN. Microglial cells survey the cerebral microenvironment and engulf cell debris. Therefore, both astrocytes and microglia are required for neuronal protection. However, astrocytes and microglial cells are not sufficient for the protection of dopaminergic neurons. Microglia and astrocytes are the main components of the innate immune system in the CNS [26, 27]. When the homeostasis of neurons, astrocytes, and microglial cells is disrupted with the administration of dopaminergic neurotoxins, such as MPTP and 6-hydroxydopamine (6-OHDA), neuroinflammatory activities mediated by activated microglia and reactive astrocytes promote neurodegeneration. Glial activation is indexed as an increase in the number and size of glial cells, as well as the induction of morphological changes.

The effects of exaggerated inflammatory responses on microglia are mainly considered deleterious [28]. Inhibition or attenuation of microglial activation is related to dopaminergic neuroprotection [29-32], with the exception of the report on IL-6 knockout mice [33]. However, the effects of reactive astrocytes are still controversial. Reactive astrocytes have been implicated in inducing oxidative stress and inflammation, therefore triggering the degeneration of dopaminergic neurons [27]. In contrast, astrocyte activation may suppress neuroinflammation and improve the resistance of dopaminergic neurons in animal models of PD. Many of the results have been revealed using gene targeting strategies in animals in combination with the neurotoxin-based PD models. Genes and their products per se are not necessarily beneficial or detrimental. In glial cells, the expressed proteome coordinately implements glial functions. The ablation of certain genes alters responses to glial activation. For example, a glial deficiency of dopamine D1 receptor or an astrocytic deficiency of dopamine D2 receptor exacerbates dopaminergic neuronal loss in MPTP-challenged mice [34, 35], whereas the activation of glial cells lacking major histocompatibility complex II (MHC II) or Lipocalin-2 (LCN2) is inhibited in mice, and the dopaminergic system is subsequently protected following the MPTP treatment $[31,32]$.

Astrocytes are activated by damaged dopaminergic neurons [32, 36] or activated microglial cells [28, 31, 37]. Moreover, reactive astrocytes also secrete soluble factors that stimulate microglial activation [10, 27]. Interestingly, the status of astrocyte activation is correlated with dopaminergic neurodegeneration in MPTP mouse models: stronger astrocyte activation is associated with more severe damage to the nigrostriatal pathway $[35,36]$; attenuated astrocyte activation is correlated with less dopaminergic neuronal loss [32]; and a lack of astrocyte activation is accompanied by nondopaminergic neurodegeneration [31].

\section{The MPTP Mouse Model of Parkinson's Disease}

Neurotoxins, such as MPTP, 6-OHDA, rotenone, paraquat, paraquat combined with manganese ethylenebisdithiocarbamate (Maneb), reserpine, and lipopolysaccharide (LPS), induce dopaminergic neurodegeneration in animals $[7,38]$. Neurotoxic effects of MPTP on humans, monkeys, rodents, zebrafish, and C. elegans have been observed. For decades, the MPTP mouse model has been the most commonly used model for elucidating damage to the nigrostriatal pathway in PD.

MPTP is systemically administrated to mice. Due to its lipophilic property, it easily crosses the BBB. Inside the brain, MPTP is converted to the intermediate 1-methyl4-phenyl-2,3-dihydropyridinium species $\left(\mathrm{MPDP}^{+}\right)$by glial monoamine oxidase-B (MAO-B) $[39,40]$. $\mathrm{MPDP}^{+}$is subsequently oxidized to the toxic metabolite 1-methyl-4phenylpyridinium $\left(\mathrm{MPP}^{+}\right)[41] . \mathrm{MPP}^{+}$is released from the astrocytes through the organic cation transporter 3 into the extracellular space, where it is taken up by the dopaminergic neurons and terminals via the plasma membrane dopamine transporter [7, 38, 42, 43]. Once $\mathrm{MPP}^{+}$accumulates in dopaminergic neurons, it induces neurotoxicity primarily by inhibiting complex I of the mitochondrial electron transport chain [44], resulting in ATP depletion and oxidative stress mediated by superoxide and NO, followed by neuronal death [3].

The inbred strain $\mathrm{C} 57 \mathrm{BL} / 6$ with high MAO-B activity is sensitive to MPTP toxicity. Following exposure to MPTP, the striatal $\mathrm{MPP}^{+}$content reaches the highest value within $2 \mathrm{~h}$ and the metabolite is cleared from the brain within $12 \mathrm{~h}$ 
[29, 45-48]. In 1995, Jackson-Lewis and Przedborski developed the acute regimen [49], which is widely adopted in the study of PD. In this case, dynamic changes in the nigrostriatal pathway are examined in the acute MPTP mouse model (four injections of MPTP- $\mathrm{HCl}$ at a dose of $18-20 \mathrm{mg} / \mathrm{kg}$ with $2 \mathrm{~h}$ intervals between injections, $1 \mathrm{mg}$ of MPTP-HCl equal to $0.826 \mathrm{mg}$ of free base MPTP). C57BL/6 mice are euthanized at 90 min to 12 h, 1 day to 9 days, and 42 days to 90 days after the last MPTP administration to analyze immediate, early, and late effects, respectively.

\section{Immediate Effects of MPTP on the Nigrostriatal Pathway}

Following an acute MPTP treatment, the striatal concentrations of DA and its metabolite DOPAC are dramatically reduced to approximately $10 \%$ of the control level at $90 \mathrm{~min}$ after injection. No changes in the striatal HVA, 5-HT, or 5HIAA levels are observed. At this time point, $67 \%$ of the THpositive $\left(\mathrm{TH}^{+}\right)$nerve fibers remained intact in the striatum. Thus, the inhibition of the enzymatic activity of TH by $\mathrm{MPP}^{+}$ is more dramatic than the degeneration of $\mathrm{TH}^{+}$terminals. Using a stereological counting method, MPTP was shown to cause a slight decrease in the number of $\mathrm{TH}^{+}$neurons in the SNpc (87\% of the control). Levels of the $\mathrm{TH}$ protein in the SN were comparable between the control and MPTPinjured mice [50]. These findings are consistent with the wellknown concept that the striatal $\mathrm{TH}^{+}$fibers have a different sensitivity to the toxin MPTP compared to $\mathrm{TH}^{+}$soma in the SNpc [51-54]. Microglial activation, which is characterized by an increase in the number of microglia and changes in morphology, was detected in the striatum at 90 min after the last MPTP injection [50] (Figure 1). Microglial activation in the striatum and the SN was confirmed $12 \mathrm{~h}$ after the acute MPTP treatment in many other studies $[55,56]$. Collectively, in the acute MPTP model, systemic injections of MPTP result in a rapid onset of neuroinflammatory responses in the striatum and the $\mathrm{SN}$, and microglial activation and proliferation precede the death of dopaminergic neurons [50, 57].

\section{Early Effects of MPTP on the Nigrostriatal Pathway}

Reductions in striatal levels of the $\mathrm{TH}$ protein and the depletion of $\mathrm{TH}^{+}$nerve fibers are observed 1 day after MPTP intoxication. According to studies performing immunohistochemical staining of the midbrain and stereological counting, the numbers of $\mathrm{TH}^{+}$neurons and Nissl-positive neurons are significantly decreased in the MPTP-lesioned SNpc. Notably, TH protein expression is not detected in the Fluoro-Jade B-positive degenerating neurons $[50,58]$. At this time point, MPTP induces widespread microglial activation in the nigrostriatal pathway, as indicated by an increase in the number and size of $\mathrm{Ibal}^{+}$microglial cells and morphological changes, such as hypertrophy [50, 59]. At 7 days after injection, a remarkable number of microglial cells were still activated in the SN of young $[35,57]$ and

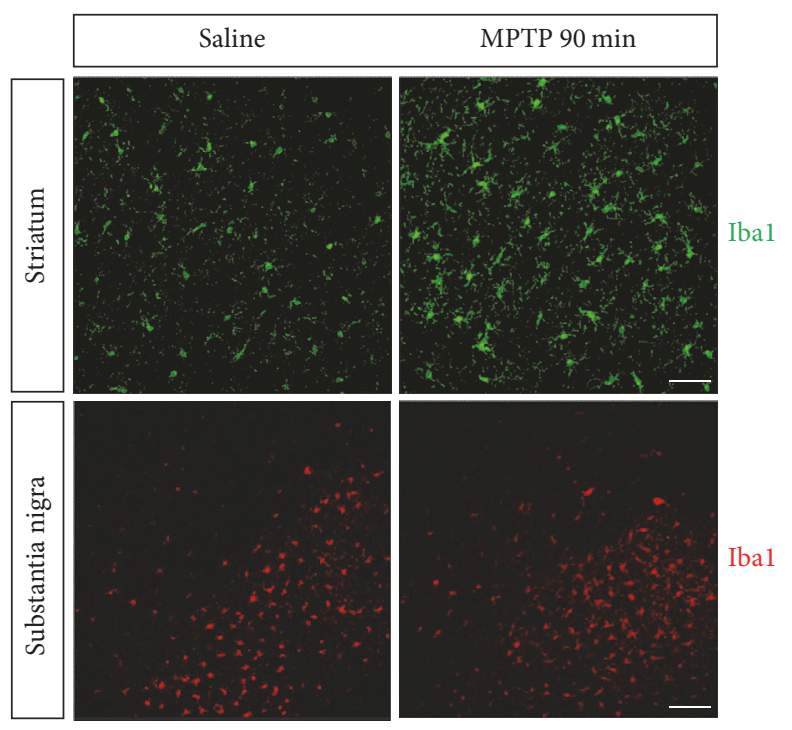

FIGURE 1: Microglial cells in the nigrostriatal pathway at $90 \mathrm{~min}$ after acute MPTP treatment. Immunofluorescence staining for Ibal (green) in the striatum (scale bar: $0.05 \mathrm{~mm}$ ) and immunofluorescence staining for Ibal (red) in the SN (scale bar: $0.1 \mathrm{~mm}$ ) were shown. This figure is adapted from Liu et al., scientific reports 5:15720 [50].

old mice [60]. One day after MPTP treatment, the striatal levels of the GFAP protein did not change, suggesting the astrocytes might still be at the resting state [58]. In another study, Muramatsu et al. observed a marked increase in the number of GFAP-positive astrocytes (exhibiting a ramified form with many fine processes) in both the striatum and the SN at 3 and 7 days after MPTP treatment [61]. Eight days after MPTP administration, the striatal levels of the GFAP protein were significantly increased [58]. Compared to the responses of microglia and neurons in the acute MPTP model, astrocytes manifest a delayed reaction, which is also involved in mediating the neuroinflammation [57].

At 9 days after the MPTP challenge, the striatal DA concentration reached $17 \%$ of the control, which was elevated compared with the value observed at $90 \mathrm{~min}$ [50]. The reductions in striatal levels of the $\mathrm{TH}$ protein, the depletion of $\mathrm{TH}^{+}$nerve fibers, and the decrease in the number of $\mathrm{TH}^{+}$neurons in the SNpc were maintained. At this time point, the activation of microglia in the nigrostriatal pathway autonomously decreased to a level similar to the resting state [50] (Figure 2).

\section{Late Effects of MPTP on the Nigrostriatal Pathway}

The entire nigrostriatal pathway undergoes a process of recovery 2-3 weeks after the acute MPTP treatment. At 42 days after the last MPTP injection, progressive striatal dopaminergic reinnervation was confirmed by DAT immunoreactivity and $\left[{ }^{3} \mathrm{H}\right]$ dopamine uptake [55]. The striatal levels of the TH protein, the intensity of $\mathrm{TH}^{+}$nerve fibers, and the number of $\mathrm{TH}^{+}$neurons in the $\mathrm{SNpc}$ were still significantly reduced 


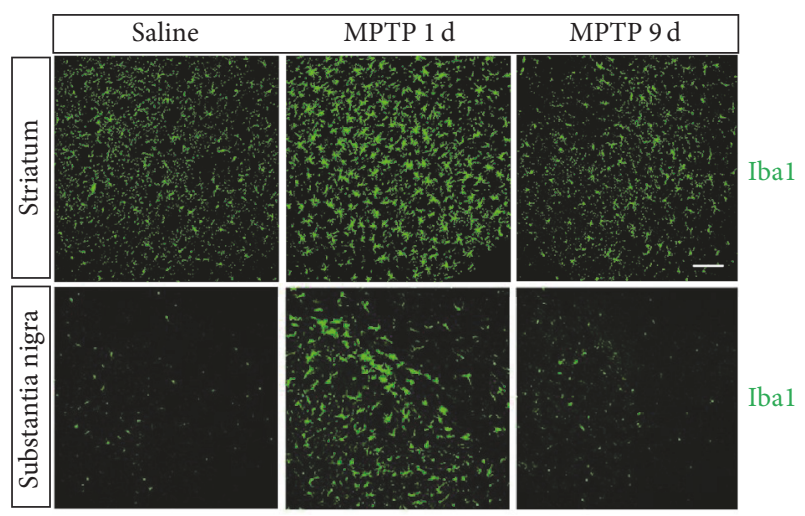

FIgURE 2: Microglial cells in the nigrostriatal pathway at 1 and 9 days after acute MPTP treatment. Immunofluorescence staining for Ibal (green) in the striatum and in the SN was shown. Scale bar: $0.1 \mathrm{~mm}$. This figure is adapted from Liu et al., scientific reports 5:15720 [50].

(unpublished data). These phenomena are maintained for 90 days after MPTP intoxication. Activated astrocytes are detected in the nigrostriatal pathway at 42 days (unpublished data), 65 days [62], and 90 days after injection (unpublished data).

\section{Dynamic Changes in the Nigrostriatal Pathway in Subacute, Subchronic, and Chronic MPTP Mouse Models}

In mice, acute MPTP treatment primarily damages the nigrostriatal dopaminergic pathway [3]. However, dopaminergic neurons die quickly and little progression in the loss of nigrostriatal DA is observed [46]. According to the study question, different MPTP regimens are applied in different studies [63]. The time course of the deleterious events and the magnitude of the lesion depend on the regimen of administration [52, 64]. Other commonly used MPTP regimens include the following:

(1) Presymptomatic PD model: an acute single MPTP application at low dose $(1 \times 10-20 \mathrm{mg} / \mathrm{kg})$

(2) Subacute PD model: repetitive subacute MPTP applications at intermediate doses $(4 \times 15-25 \mathrm{mg} / \mathrm{kg}$ at 6 or $12 \mathrm{~h}$ intervals within two days)

(3) Subchronic PD model: daily MPTP injections at doses of approximately $20-30 \mathrm{mg} / \mathrm{kg}$ for up to $4-5$ consecutive days

(4) Progressive chronic PD model: daily injections of low doses $(4 \mathrm{mg} / \mathrm{kg}$ ) of MPTP over 20 days.

In the subacute $\mathrm{PD}$ regimen, adult male $\mathrm{C} 57 \mathrm{BL} / 6$ mice were challenged with MPTP (24 mg/kg, every $12 \mathrm{~h}$ for $2 \mathrm{~d}$ ). Mice were euthanized at different time points after the last dose. TH protein expression in both the striatum and the SN is noticeably decreased. According to the statistical analysis, the levels of $\mathrm{TH}$ protein in the striatum at 18,36 , and $72 \mathrm{~h}$ after injection were $38 \%, 45 \%$, and $31 \%$ of the saline group, respectively, and the levels in the SN were $60 \%, 38 \%$, and
$67 \%$ of the control, respectively [65]. The dose of MPTP administered to 12 -month-old mice was reduced to $15 \mathrm{mg} / \mathrm{kg}$. Four weeks after the MPTP treatment, the level of striatal TH protein decreased by $59 \%$ compared with saline-treated mice, whereas the striatal DA concentration after the MPTP treatment decreased by 79\%. Approximately 63\% of dopaminergic neurons in the SN were lost following the MPTP treatment. The striatal GFAP level increased by 3.7-fold at 3 days after MPTP administration, and this level was maintained for 4 weeks in old mice [36].

The different responses observed in young and old mice have been repeatedly measured, but with some variation. The dosages of MPTP and the age are likely the main determining factors. Young (9-12 weeks old) and old (11-13 months old) mice were intraperitoneally injected with MPTP twice a day at $12 \mathrm{~h}$ intervals for 2 days. The dosage of MPTP was $20 \mathrm{mg} / \mathrm{kg}$ for young mice and $15 \mathrm{mg} / \mathrm{kg}$ for old animals. One or 3 days after the last MPTP injection, MPTP elicited an approximately $90 \%$ (1 day) to $55 \%$ (3 days) decrease in the striatal levels of the $\mathrm{TH}$ protein in young mice. In old mice, MPTP induced a nearly $70 \%$ (1 day or 3 days) decrease. On the 8 th day, MPTP elicited a $57 \%$ decrease in the striatal levels of the TH protein. Approximately $80 \%$ and $70 \%$ losses of $\mathrm{TH}^{+}$fibers were observed in young mice 1 day or 3 days after MPTP intoxication. Meanwhile, MPTP caused an approximately $66 \%$ (1 day) to $58 \%$ (3 days) decrease in the density of $\mathrm{TH}^{+}$fibers in old mice. In the SN, MPTP elicited approximately $41 \%$ (1 day) to $38 \%$ (3 days) and 50\% (1 day) to $53 \%$ (3 days) loss of $\mathrm{TH}^{+}$neurons in young and old mice, respectively. Based on electron microscopic analyses, MPTP induced a significant increase in the number of structurally altered mitochondria in the SNpc neurons of young mice at 3 days after injection; these alterations comprised numerous vacuoles and fragmented cristae. According to the quantitative analysis, the SNpc neurons exhibited a significantly higher percentage of damaged mitochondria (51.3\%) [54]. In a study with cHS4I-hIL-1 $\beta$ P-Luc transgenic mice, in which the expression of luciferase reporter gene is controlled by the human $I L-1 \beta$ gene promoter [66], both old male and female mice were monitored following subacute MPTP intoxication. MPTP induced elevated expression of the IL- $1 \beta$ transcript in the cortex, striatum, and ventral midbrain at $2 \mathrm{~h}$ after treatment. Luciferase expression was significantly elevated at 2, 8, 32, and $49 \mathrm{~h}$ in MPTP-treated male mice, the inducible signals peaked at $8 \mathrm{~h}$. The old female mice showed a marked increase in luciferase expression at 4 and $26 \mathrm{~h}$ after MPTP administration. At $96 \mathrm{~h}$ after the last MPTP injection, striatal levels of the GFAP protein were robustly increased. As expected, MPTP elicited less dopaminergic toxicity in old female than in male mice [67].

In the subchronic $P D$ regimen $(30 \mathrm{mg} / \mathrm{kg} /$ day for 5 consecutive days), MPTP induced the depletion of more than $90 \%$ of the $\mathrm{TH}$ protein in the striatum and reduced the number of $\mathrm{TH}^{+}$neurons in the SNpc by $30 \%$ at $24 \mathrm{~h}$ after injection. Notably, MPTP administration significantly increased the expression of $\alpha$-synuclein in the striatum ([68] and unpublished data). The observation of reactive gliosis is also compelling in this model. A significantly higher number of Iba 1-positive cells were observed in the SNpc at 1 day [31] 


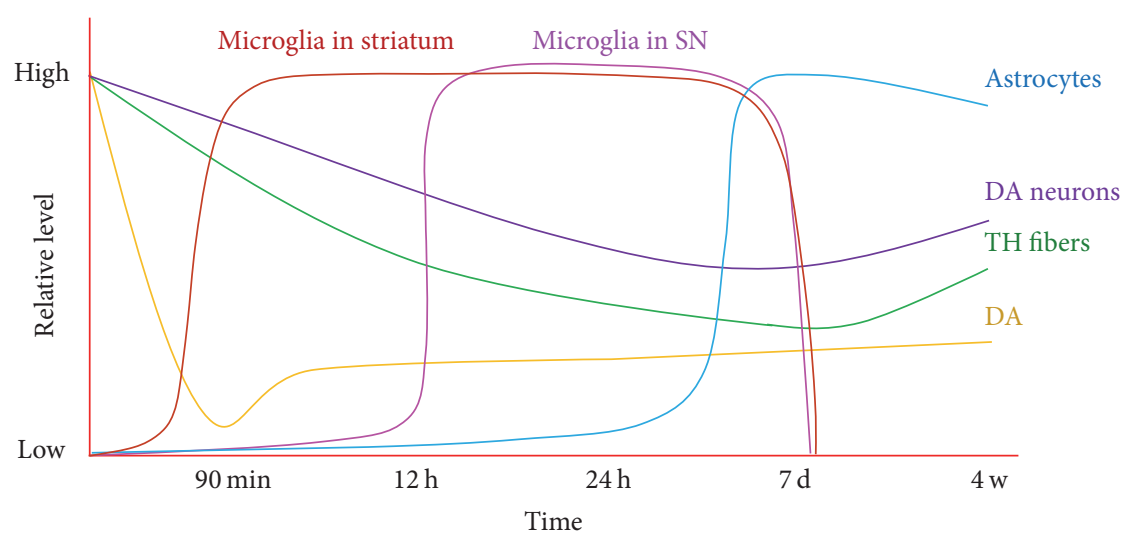

FIGURE 3: Dynamic changes in the nigrostriatal pathway in the acute MPTP mouse model of Parkinson's disease.

or 2 days [30], after MPTP treatment. However, at 2 days after injection, astrogliosis is not consistently detected [30, 31]. Three weeks after the injection, the striatal DA level was reduced to $17 \%$ of the control level by the MPTP treatment [31], whereas $42 \%$ of the $\mathrm{TH}^{+}$neurons in the $\mathrm{SNpc}$ and $14 \%$ of $\mathrm{TH}^{+}$fibers (by density assay) in the striatum were preserved and the astroglial cell count increased [30].

In summary, the specific and reproducible neurotoxic effect on the nigrostriatal system are a strength of MPTP induced PD models. However, MPTP mouse models exhibit an apparent lack of Lewy body-like inclusions bodies in the midbrain $[46,69]$. Here, the communication between the CNS and immune system, which also contributes to the progression of $\mathrm{PD}$, should be mentioned. Peripheral immune cells play an important role during the course of neuroinflammation in the mouse MPTP models, with reports of $\mathrm{CD}^{+}$and $\mathrm{CD}^{+}{ }^{+}$-lymphocytes infiltrating the SN [70].

\section{Conclusions}

The nigrostriatal pathway plays an important role in regulating the functions of the basal ganglion. Under physiological conditions, neurons, astrocytes, and microglia support each other and maintain a triple "win-win" relationship. When exposed to environmental or endogenous toxins, which may be combined with genetic susceptibility, this circuit is damaged, causing motor symptoms in patients with PD. The strengths and limitations of MPTP mouse models of PD are both remarkable. Microglial activation precedes the degeneration of dopaminergic neurons and astrocyte activation in the nigrostriatal pathway of the acute MPTP mouse model. The dynamic changes observed in the nigrostriatal pathway are summarized in Figure 3. A better understanding of the time course of pathophysiological events will benefit studies developing interventions or therapeutic strategies for PD.

\section{Conflicts of Interest}

The authors have no conflicts of interest to declare regarding the publication of this paper.

\section{Authors' Contributions}

Dongping Huang and Jing $\mathrm{Xu}$ contributed equally to this paper.

\section{Acknowledgments}

This work was supported by grants from the National Natural Science Foundation of China (31671043, 81371412, and 81400992) and the Open Project of State Key Laboratory of Medical Neurobiology (SKLMN2015005).

\section{References}

[1] R. L. Mosley, J. A. Hutter-Saunders, D. K. Stone, and H. E. Gendelman, "Inflammation and adaptive immunity in Parkinson's disease," Cold Spring Harbor Perspectives in Medicine, vol. 2, no. $1,2012$.

[2] T. M. Dawson, H. S. Ko, and V. L. Dawson, "Genetic animal models of Parkinson's disease," Neuron, vol. 66, no. 5, pp. 646661,2010

[3] W. Dauer and S. Przedborski, "Parkinson's disease: mechanisms and models," Neuron, vol. 39, no. 6, pp. 889-909, 2003.

[4] L. S. Forno, "Neuropathology of Parkinson's disease," Journal of Neuropathology and Experimental Neurology, vol. 55, no. 3, pp. 259-272, 1996.

[5] L. Drew, “Two hundred steps," Nature, vol. 538, no. 7626, pp. S2-S3, 2016.

[6] S. Deweerdt, "Parkinson's disease: 4 big questions," Nature, vol. 538, no. 7626, p. S17, 2016.

[7] K. Tieu, "A guide to neurotoxic animal models of Parkinson's disease," Cold Spring Harbor perspectives in medicine, vol. 1, no. 1, p. a009316, 2011.

[8] G. C. Davis, A. C. Williams, S. P. Markey et al., "Chronic parkinsonism secondary to intravenous injection of meperidine analogues," Psychiatry Research, vol. 1, no. 3, pp. 249-254, 1979.

[9] J. W. Langston, P. Ballard, J. W. Tetrud, and I. Irwin, "Chronic parkinsonism in humans due to a product of meperidineanalog synthesis," Science, vol. 219, no. 4587, pp. 979-980, 1983.

[10] K. Saijo and C. K. Glass, "Microglial cell origin and phenotypes in health and disease," Nature Reviews Immunology, vol. 11, no. 11, pp. 775-787, 2011. 
[11] F. Alliot, I. Godin, and B. Pessac, "Microglia derive from progenitors, originating from the yolk sac, and which proliferate in the brain," Developmental Brain Research, vol. 117, no. 2, pp. 145-152, 1999.

[12] M. R. P. Elmore, A. R. Najafi, M. A. Koike et al., "Colonystimulating factor 1 receptor signaling is necessary for microglia viability, unmasking a microglia progenitor cell in the adult brain," Neuron, vol. 82, pp. 380-397, 2014.

[13] J. Bruttger, K. Karram, S. Wörtge et al., "Genetic cell ablation reveals clusters of local self-renewing microglia in the mammalian central nervous system," Immunity, vol. 43, no. 1, pp. 92107, 2015.

[14] S. Jäkel and L. Dimou, "Glial cells and their function in the adult brain: a journey through the history of their ablation," Frontiers in Cellular Neuroscience, vol. 11, 24 pages, 2017.

[15] L. J. Lawson, V. H. Perry, P. Dri, and S. Gordon, "Heterogeneity in the distribution and morphology of microglia in the normal adult mouse brain," Neuroscience, vol. 39, no. 1, pp. 151-170, 1990.

[16] H. Kettenmann, U. K. Hanisch, M. Noda, and A. Verkhratsky, "Physiology of microglia," Physiological Reviews, vol. 91, no. 2, pp. 461-553, 2011.

[17] A. Suzumura, "Neuron-microglia interaction in neuroinflammation," Current Protein and Peptide Science, vol. 14, no. 1, pp. 16-20, 2013.

[18] P. L. McGeer, S. Itagaki, B. E. Boyes, and E. G. McGeer, "Reactive microglia are positive for HLA-DR in the substantia nigra of Parkinson's and Alzheimer's disease brains," Neurology, vol. 38, no. 8, pp. 1285-1291, 1988.

[19] Q. Wang, Y. Liu, and J. Zhou, "Neuroinflammation in Parkinson's disease and its potential as therapeutic target," Neurodegeneration, vol. 4, article 19, 2015.

[20] S. Lee, J.-Y. Park, W.-H. Lee et al., "Lipocalin-2 is an autocrine mediator of reactive astrocytosis," Journal of Neuroscience, vol. 29, no. 1, pp. 234-249, 2009.

[21] H. K. Kimelberg and M. Nedergaard, "Functions of astrocytes and their potential as therapeutic targets," Neurotherapeutics, vol. 7, no. 4, pp. 338-353, 2010.

[22] R. Srinivasan, T.-Y. Lu, H. Chai et al., "New Transgenic Mouse Lines for Selectively Targeting Astrocytes and Studying Calcium Signals in Astrocyte Processes In Situ and In Vivo," Neuron, vol. 92, no. 6, pp. 1181-1195, 2016.

[23] H. M. Jahn, A. Scheller, and F. Kirchhoff, "Genetic control of astrocyte function in neural circuits," Frontiers in Cellular Neuroscience, vol. 9, no. AUGUST, article no. 310, 2015.

[24] J. W. Langston, L. S. Forno, J. Tetrud, A. G. Reeves, J. A. Kaplan, and D. Karluk, "Evidence of active nerve cell degeneration in the substantia nigra of humans years after 1-methyl-4-phenyl1,2,3,6-tetrahydropyridine exposure," Annals of Neurology, vol. 46, no. 4, pp. 598-605, 1999.

[25] T. Yamada, T. Kawamata, D. G. Walker, and P. L. McGeer, "Vimentin immunoreactivity in normal and pathological human brain tissue," Acta Neuropathologica, vol. 84, no. 2, pp. 157162, 1992.

[26] M. C. O. Rodrigues, P. R. Sanberg, L. E. Cruz, and S. GarbuzovaDavis, "The innate and adaptive immunological aspects in neurodegenerative diseases," Journal of Neuroimmunology, vol. 269, no. 1-2, pp. 1-8, 2014.

[27] C. K. Glass, K. Saijo, B. Winner, M. C. Marchetto, and F. H. Gage, "Mechanisms underlying inflammation in neurodegeneration," Cell, vol. 140, no. 6, pp. 918-934, 2010.
[28] K. Saijo, B. Winner, C. T. Carson et al., "A Nurr1/CoREST pathway in microglia and astrocytes protects dopaminergic neurons from inflammation-induced death," Cell, vol. 137, no. 1, pp. 47-59, 2009.

[29] M. Moon, H. G. Kim, L. Hwang et al., "Blockade of microglial activation is neuroprotective in the 1-methyl-4-phenyl-1,2,3,6tetrahydropyridine mouse model of Parkinson disease," Journal of Neuroscience, vol. 22, no. 5, pp. 1763-1771, 2002.

[30] K. Sathe, W. Maetzler, J. D. Lang et al., "S100B is increased in Parkinson's disease and ablation protects against MPTPinduced toxicity through the RAGE and TNF- $\alpha$ pathway," Brain, vol. 135, no. 11, pp. 3336-3347, 2012.

[31] H. L. Martin, M. Santoro, S. Mustafa, G. Riedel, J. V. Forrester, and P. Teismann, "Evidence for a role of adaptive immune response in the disease pathogenesis of the MPTP mouse model of Parkinson's disease," Glia, vol. 64, no. 3, pp. 386-395, 2016.

[32] B.-W. Kim, K. H. Jeong, J.-H. Kim et al., "Pathogenic upregulation of glial lipocalin-2 in the parkinsonian dopaminergic system," Journal of Neuroscience, vol. 36, no. 20, pp. 5608-5622, 2016.

[33] H. Cardenas and L. M. Bolin, "Compromised reactive microgliosis in MPTP-lesioned IL-6 KO mice," Brain Research, vol. 985, no. 1, pp. 89-97, 2003.

[34] Y. Yan, W. Jiang, L. Liu et al., "Dopamine controls systemic inflammation through inhibition of NLRP3 inflammasome," Cell, vol. 160, no. 1-2, pp. 62-73, 2015.

[35] W. Shao, S.-Z. Zhang, M. Tang et al., "Suppression of neuroinflammation by astrocytic dopamine D2 receptors via $\alpha \mathrm{b}$ crystallin," Nature, vol. 494, no. 7435, pp. 90-94, 2013.

[36] M. Yu, H. Suo, M. Liu et al., "NRSF/REST neuronal deficient mice are more vulnerable to the neurotoxin MPTP," Neurobiology of Aging, vol. 34, no. 3, pp. 916-927, 2013.

[37] S. A. Liddelow, K. A. Guttenplan, L. E. Clarke et al., "Neurotoxic reactive astrocytes are induced by activated microglia," Nature, vol. 541, no. 7638, pp. 481-487, 2017.

[38] J. Bové and C. Perier, “Neurotoxin-based models of Parkinson's disease," Neuroscience, vol. 211, pp. 51-76, 2012.

[39] R. E. Heikkila, L. Manzino, F. S. Cabbat, and R. C. Duvoisin, "Protection against the dopaminergic neurotoxicity of 1-methyl4-phenyl-1,2, 5,6-tetrahydropyridine by monoamine oxidase inhibitors," Nature, vol. 311, no. 5985, pp. 467-469, 1984.

[40] K. Chiba, A. Trevor, and N. Castagnoli Jr., "Metabolism of the neurotoxic tertiary amine, MPTP, by brain monoamine oxidase," Biochemical and Biophysical Research Communications, vol. 120 , no. 2, pp. 574-578, 1984.

[41] R. Heikkila, A. Hess, and R. Duvoisin, "Dopaminergic neurotoxicity of 1-methyl-4-phenyl-1,2,5,6-tetrahydropyridine in mice," Science, vol. 224, no. 4656, pp. 1451-1453, 1984.

[42] S. J. Choi, A. Panhelainen, Y. Schmitz et al., "Changes in neuronal dopamine homeostasis following 1-methyl-4-phenylpyridinium (MPP+) exposure," Journal of Biological Chemistry, vol. 290, no. 11, pp. 6799-6809, 2015.

[43] J. A. Javitch, R. J. D’Amato, S. M. Strittmatter, and S. H. Snyder, "Parkinsonism-inducing neurotoxin, N-methyl-4-phenyl1,2,3,6 -tetrahydropyridine: uptake of the metabolite N-methyl4-phenylpyridine by dopamine neurons explains selective toxicity," Proceedings of the National Academy of Sciences of the United States of America, vol. 82, no. 7, pp. 2173-2177, 1985. 
[44] W. J. Nicklas, I. Vyas, and R. E. Heikkila, "Inhibition of NADHlinked oxidation in brain mitochondria by 1-methyl-4-phenylpyridine, a metabolite of the neurotoxin, 1-methyl-4-phenyl1,2,5,6-tetrahydropyridine," Life Sciences, vol. 36, no. 26, pp. 2503-2508, 1985.

[45] V. Jackson-Lewis and S. Przedborski, "Protocol for the MPTP mouse model of Parkinson's disease," Nature Protocols, vol. 2, no. 1, pp. 141-151, 2007.

[46] G. E. Meredith and D. J. Rademacher, "MPTP mouse models of Parkinson's disease: an update," Journal of Parkinson's Disease, vol. 1, no. 1, pp. 19-33, 2011.

[47] T. Kawasaki, Y. Ago, T. Kitao et al., "A neuroprotective agent, T817MA (1-3-[2-(1-benzothiophen-5-yl)ethoxy]propyl azetidin3-ol maleate), prevents 1-methyl-4-phenyl-1,2,3,6-tetrahydropyridine-induced neurotoxicity in mice," Neuropharmacology, vol. 55, no. 5, pp. 654-660, 2008.

[48] Y. C. Chung, S. R. Kim, J. Park et al., "Fluoxetine prevents MPTP-induced loss of dopaminergic neurons by inhibiting microglial activation," Neuropharmacology, vol. 60, no. 6, pp. 963-974, 2011.

[49] V. Jackson-Lewis, M. Jakowec, R. E. Burke, and S. Przedborski, "Time course and morphology of dopaminergic neuronal death caused by the neurotoxin 1-methyl-4-phenyl-1,2,3,6-tetrahydropyridine," Neurodegeneration, vol. 4, no. 3, pp. 257-269, 1995.

[50] J. Liu, D. Huang, J. Xu et al., “Tiagabine Protects Dopaminergic Neurons against Neurotoxins by Inhibiting Microglial Activation," Scientific Reports, vol. 5, Article ID 15720, 2015.

[51] P. Teismann, K. Tieu, D. K. Choi et al., "Cyclooxygenase2 is instrumental in Parkinson's disease neurodegeneration," Proceedings of the National Academy of Sciences of the United States of America, vol. 100, no. 9, pp. 5473-5478, 2003.

[52] C. Gibrat, M. Saint-Pierre, M. Bousquet, D. Lévesque, C. Rouillard, and F. Cicchetti, "Differences between subacute and chronic MPTP mice models: investigation of dopaminergic neuronal degeneration and $\alpha$-synuclein inclusions," Journal of Neurochemistry, vol. 109, no. 5, pp. 1469-1482, 2009.

[53] G. Costa, L. Frau, J. Wardas, A. Pinna, A. Plumitallo, and M. Morelli, "MPTP-induced dopamine neuron degeneration and glia activation is potentiated in MDMA-pretreated mice," Movement Disorders, vol. 28, no. 14, pp. 1957-1965, 2013.

[54] M. Bian, J. Liu, X. Hong et al., "Overexpression of parkin ameliorates dopaminergic neurodegeneration induced by 1- methyl4-phenyl-1,2,3,6-tetrahydropyridine in mice," PLoS ONE, vol. 7, no. 6, Article ID e39953, 2012.

[55] F. L'Episcopo, C. Tirolo, N. Testa et al., "Plasticity of subventricular zone neuroprogenitors in MPTP (1-Methyl-4-Phenyl1,2,3,6-tetrahydropyridine) mouse model of Parkinson's disease involves cross talk between inflammatory and $\mathrm{Wnt} / \beta$-catenin signaling pathways: functional consequences for neuroprotection and repair," Journal of Neuroscience, vol. 32, no. 6, pp. 20622085, 2012.

[56] T. Furuya, H. Hayakawa, M. Yamada et al., "Caspase-11 mediates inflammatory dopaminergic cell death in the 1-methyl-4phenyl-1,2,3,6-tetrahydropyridine mouse model of Parkinson's disease," Journal of Neuroscience, vol. 24, no. 8, pp. 1865-1872, 2004.

[57] M. Kohutnicka, E. Lewandowska, I. Kurkowska-Jastrzebska, A. Członkowski, and A. Członkowska, "Microglial and astrocytic involvement in a murine model of Parkinson's disease induced by 1-methyl-4-phenyl-1,2,3,6-tetrahydropyridine (MPTP)," Immunopharmacology, vol. 39, no. 3, pp. 167-180, 1998.
[58] H. Suo, P. Wang, J. Tong et al., "NRSF is an essential mediator for the neuroprotection of trichostatin A in the MPTP mouse model of Parkinson's disease," Neuropharmacology, vol. 99, pp. 67-78, 2015.

[59] M. Jin, B. W. Kim, S. Koppula et al., "Molecular effects of activated BV-2 microglia by mitochondrial toxin 1-methyl-4phenylpyridinium," NeuroToxicology, vol. 33, no. 2, pp. 147-155, 2012.

[60] J. Xu, L. Bu, L. Huang et al., "Heart failure having little effect on the progression of Parkinson's disease: Direct evidence from mouse model," International Journal of Cardiology, vol. 177, no. 2, pp. 683-689, 2014.

[61] Y. Muramatsu, R. Kurosaki, H. Watanabe et al., "Expression of S-100 protein is related to neuronal damage in MPTP-treated mice," GLIA, vol. 42, no. 3, pp. 307-313, 2003.

[62] F. L'Episcopo, C. Tirolo, N. Testa et al., "Wnt/ $\beta$-catenin signaling is required to rescue midbrain dopaminergic progenitors and promote neurorepair in ageing mouse model of Parkinson's disease," Stem Cells, vol. 32, no. 8, pp. 2147-2163, 2014.

[63] V. Machado, T. Zöller, A. Attaai, and B. Spittau, "Microgliamediated neuroinflammation and neurotrophic factor-induced protection in the MPTP mouse model of parkinson's diseaselessons from transgenic mice," International Journal of Molecular Sciences, vol. 17, no. 2, article no. 151, 2016.

[64] S. Przedborski and M. Vila, "The 1-methyl-4-phenyl-1,2,3,6tetrahydropyridine mouse model: a tool to explore the pathogenesis of Parkinson's disease," Annals of the New York Academy of Sciences, vol. 991, pp. 189-198, 2003.

[65] M. Bian, M. Yu, S. Yang et al., "Expression of Cbl-interacting protein of $85 \mathrm{kDa}$ in MPTP mouse model of Parkinson's disease and 1-methyl-4-phenyl-pyridinium ion-treated dopaminergic SH-SY5Y cells," Acta Biochimica et Biophysica Sinica, vol. 40, no. 6, pp. 505-512, 2008.

[66] L. Li, Z. Fei, J. Ren et al., "Functional imaging of interleukin 1 beta expression in inflammatory process using bioluminescence imaging in transgenic mice," BMC Immunology, vol. 9, article no. 49, 2008.

[67] M.-J. Bian, L.-M. Li, M. Yu, J. Fei, and F. Huang, "Elevated interleukin-1 $\beta$ induced by 1-methyl-4-phenyl-1,2,3,6-tetrahydropyridine aggravating dopaminergic neurodegeneration in old male mice," Brain Research, vol. 1302, pp. 256-264, 2009.

[68] M. Xia, M. Bian, Q. Yu et al., "Cold water stress attenuates dopaminergic neurotoxicity induced by 1-methyl-4-phenyl1,2,3,6-tetrahydropyridine in mice," Acta Biochimica et Biophysica Sinica, vol. 43, no. 6, pp. 448-454, 2011.

[69] D. Alvarez-Fischer, S. Guerreiro, S. Hunot et al., "Modelling Parkinson-like neurodegeneration via osmotic minipump delivery of MPTP and probenecid," Journal of Neurochemistry, vol. 107, no. 3, pp. 701-711, 2008.

[70] I. Kurkowska-Jastrzebska, A. Wrońska, M. Kohutnicka, A. Czlonkowski, and A. Czlonkowska, "The inflammatory reaction following 1-methyl-4-phenyl-1,2,3,6- tetrahydropyridine intoxication in mouse," Experimental Neurology, vol. 156, no. 1, pp. 50-61, 1999. 


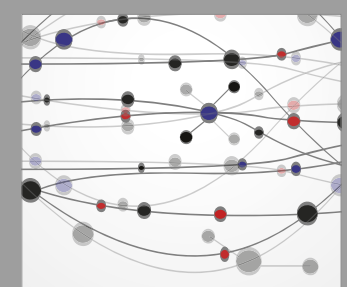

The Scientific World Journal
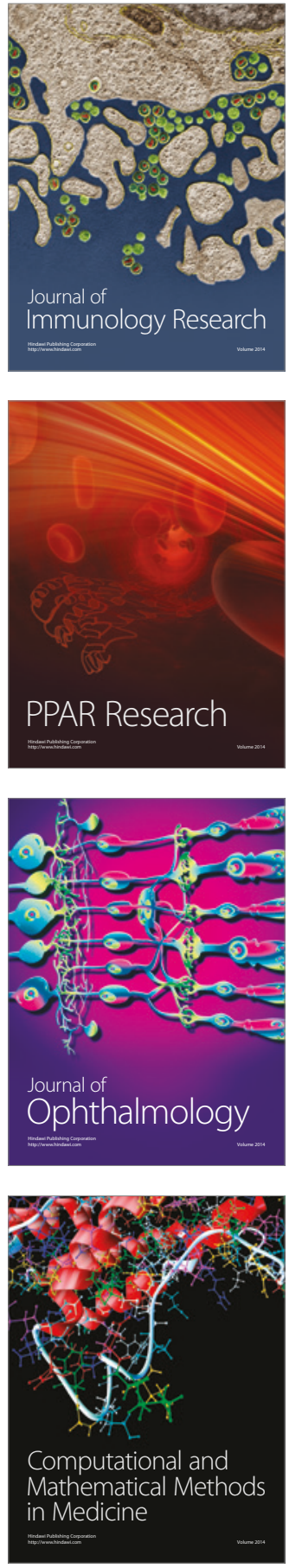

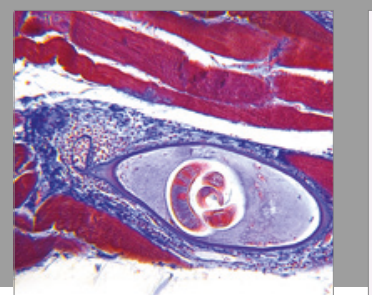

Gastroenterology Research and Practice
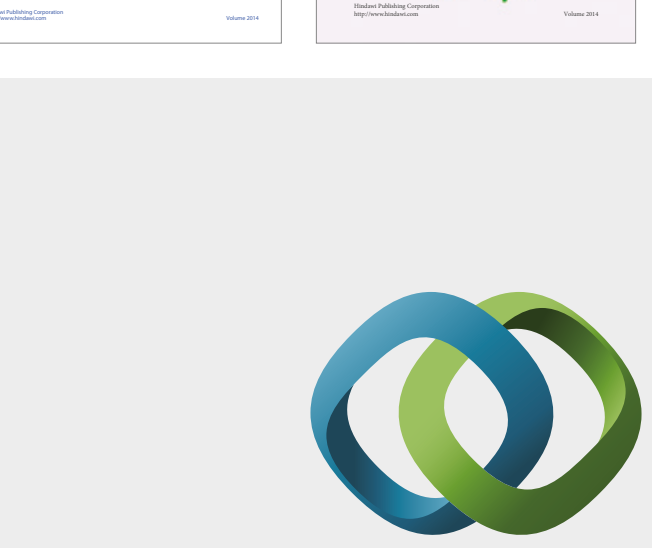

\section{Hindawi}

Submit your manuscripts at

https://www.hindawi.com
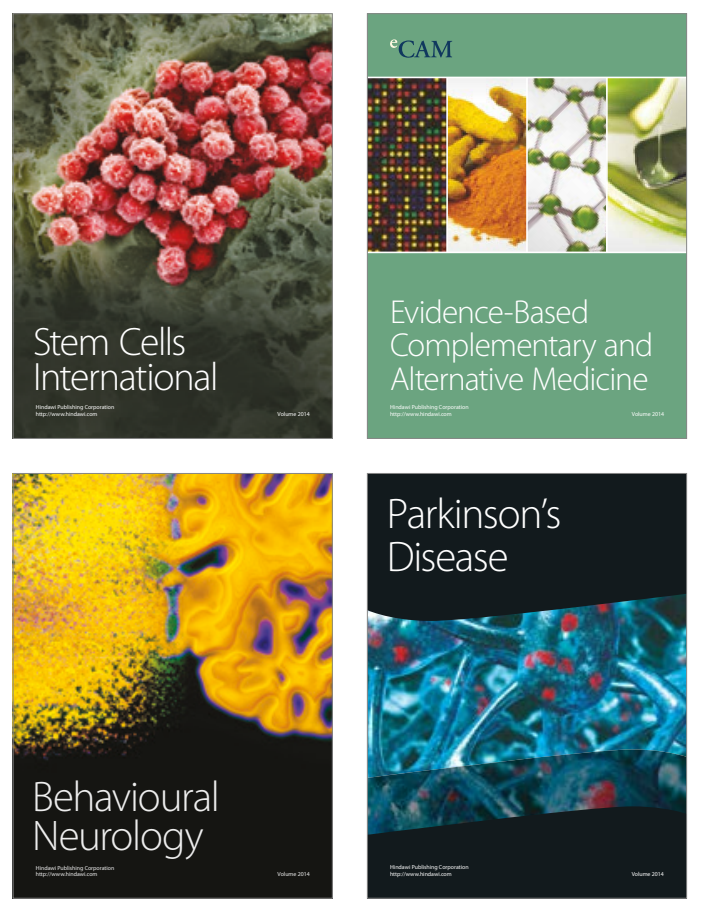
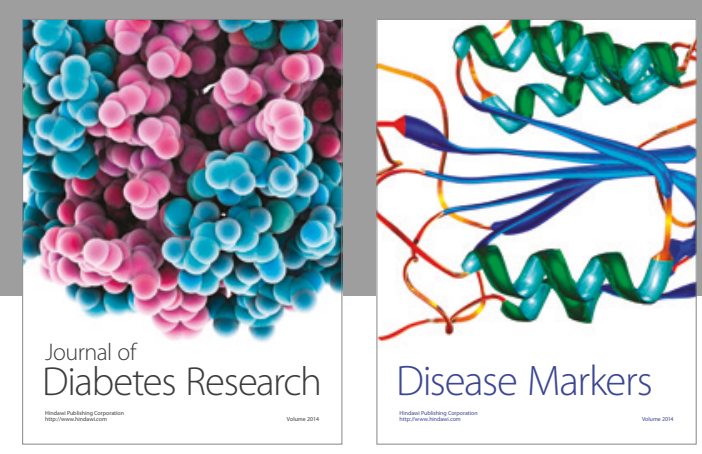

Disease Markers
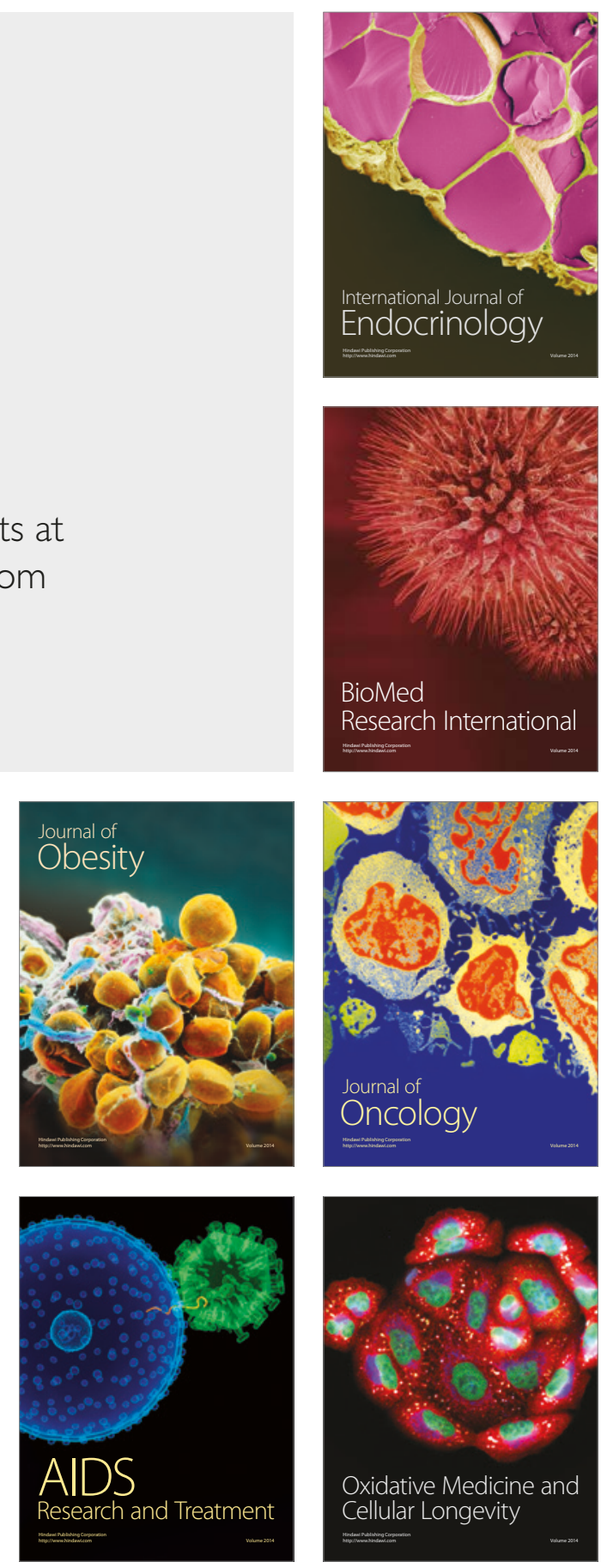